The University of San Francisco

USF Scholarship: a digital repository @ Gleeson Library |

Geschke Center

Finance

School of Management

2013

\title{
Uses and Misuses of the Black-Litterman Model in Portfolio Construction
}

\author{
Ludwig B. Chincarini \\ University of San Francisco, lbchincarini@usfca.edu \\ Daehwan Kim
}

Follow this and additional works at: http://repository.usfca.edu/fe

Part of the Finance and Financial Management Commons

\section{Recommended Citation}

Ludwig B. Chincarini, Daehwan Kim. Uses and Misuses of the Black-Litterman Model in Portfolio Construction. Journal of Mathematical Finance Vol.3 No.1A, Pub. Date: March 29, 2013

This Article is brought to you for free and open access by the School of Management at USF Scholarship: a digital repository @ Gleeson Library | Geschke Center. It has been accepted for inclusion in Finance by an authorized administrator of USF Scholarship: a digital repository @ Gleeson

Library | Geschke Center. For more information, please contact repository@usfca.edu. 


\title{
Uses and Misuses of the Black-Litterman Model in Portfolio Construction"
}

\author{
Ludwig B. Chincarini ${ }^{1}$, Daehwan Kim ${ }^{2}$ \\ ${ }^{1}$ School of Management, University of San Francisco, San Francisco, USA \\ ${ }^{2}$ Department of Economics, Konkuk University, Seoul, Korea \\ Email: chincarinil@hotmail.com, kimdaewan@hotmail.com
}

Received December 19, 2012; revised January 22, 2013; accepted February 6, 2013

\begin{abstract}
The Black-Litterman model has gained popularity in applications in the area of quantitative equity portfolio management. Unfortunately, many recent applications of the Black-Litterman to novel aspects of quantitative portfolio management have neglected the rigor of the original Black-Litterman modelling. In this article, we critically examine some of these applications from a Bayesian perspective. We identify three reasons why these applications may create losses to investors. These three reasons are: 1) Using a prior without "anchoring" the prior to an equilibrium model; 2) Using a prior and an equilibrium model that conflict with one another; and 3) Ignoring the implications of the estimation error of the variance-covariance matrix. We also quantify the loss first analytically and also numerically based on historical data on 10 major world stock market indices. Our conservative estimate of the loss is around a $1 \%$ reduction in the annualized return of the portfolio.
\end{abstract}

Keywords: Black-Litterman; Bayes Rule; Portfolio Construction; Econometric Techniques; Simulations

\section{Introduction}

The Black-Litterman model is a powerful tool in the portfolio construction process. It has gained popularity among practitioners for the past two decades, and its applications to various aspects of the portfolio construction process have been discussed in the literature. We believe, however, that some of the applications adopted by practitioners and discussed in the literature deserve more critical examination. In this paper, we will highlight the uses of the Black Litterman model that we find problematic, or at least lacking the vigor of the original formulation of the Black-Litterman model. We will present numerical examples to make our case stronger and, where appropriate, will propose alternative approaches.

Black and Litterman [1,2] saw two strengths of their approach:

1) The subjective views of the investors can be easily incorporated in the portfolio construction process.

2) The Black-Litterman mean-variance optimization does not produce unreasonable solutions, as the standard mean-variance framework does.

The first of these two comes from the feature of the model that investors' subjective views are expressed as linear combinations of expected returns of assets, rather

\footnotetext{
*We would like to especially thank Josh Ruben for research assistance. We also thank Frank Fabozzi for comments.
}

than as expected returns of individual assets. That is, the subjective view need not be an exact value of the expected return of an individual asset, but rather can be expressed as the expected return of two assets or more in relation to each other. This type of formulation is easier for investors to apply. The second strength comes from the model's feature that the investors' subjective views are combined with an equilibrium model that tilts the portfolio weights away from the market capitalization weights based on the relative uncertainty in the investor's views. This anchors the portfolio weights towards the implied market capitalization weights, thus not allowing for extreme weights due to differences in expected returns.

Out of these two features, the first feature is less essential to the Black-Litterman model. It is relatively easy to come up with an alternative way of specifying investors' subjective views. ${ }^{1}$ The spirit of the Black Litterman model can be retained from a variety of subjective prior specifications. The second feature, however, is much more significant. An attempt to modify this feature of the

\footnotetext{
${ }^{1}$ The Black Litterman model allows an expression of subjective views such as "the sum of asset A return and asset B return will be positive." That is, it allows the investor to make a statement on a linear combination of many asset returns. If this type of statement is not allowed, one may have to be more explicit. An example is: "asset a return is likely to be around $10 \%$ while asset B return is likely to be around $5 \%$."
} 
model is likely to introduce inconsistencies. The reason is that there is really only one right way to combine investors' subjective views with a given model, and there is no other correct alternative. One may choose different views or different models, but once the views and a model are chosen, there is only one way to combine them according to Bayes' rule.

Some applications of the Black-Litterman model by other authors have attempted to modify the second feature of the model, and, in doing so, they have lost the mathematical rigor of the original Black-Litterman model. Other applications have simply ignored the nature of the subjective view and mixed it up with the model in an inconsistent manner.

In this paper we discuss three representative (mis-) uses of the Black-Litterman model in portfolio construction. We will attempt to quantify the possible losses created by the misuse of the model and, where appropriate, propose an alternative. ${ }^{2}$ The first application we consider was discussed in Jones, Lim, and Zangari [4]. ${ }^{3}$ We will argue that their approach could create a loss to investors because the prior is not "anchored" to an equilibrium model. More specifically, the estimate of the mean return from the model is not included in the process, making the prediction of the mean return less than optimal. The second application we consider was discussed in Fabbozi, Forcardi, and Kolm [5]. We will argue that their approach could create losses to investors if the prior and the equilibrium model conflict each other. The third application we consider is the so called reverse optimization, which is quite popular among practitioners. We will argue that this reverse optimization could have surprisingly large errors in the resulting mean estimates.

In this article, we take a Bayesian perspective. A Bayesian perspective allows us to quantify the losses of investors without too many complications in the analysis. We borrow the framework of Satchell and Scowcroft [6], and extend it so that investors' loss can be discussed.

The paper is organized as follows: Section 2 discusses the use of the Black-Litterman technique without an equilibrium model, and the potential losses associated with that methodology; Section 3 discusses the use of the Black-Litterman model with data based priors that conflict with the model, and the loss associated with that methodology; Section 4 discusses the use of the BlackLitterman approach as a reverse optimization and the implication of using an estimated variance-covariance matrix; and Section 5 concludes the paper.

\footnotetext{
${ }^{2}$ Chincarini and Kim [3] examined other situations in which improper portfolio construction led to utility losses to the investor.

${ }^{3}$ We are not implying that the researchers we cite were unaware of the problems we are raising. In fact, the applications we discuss were not the main point of their articles, which explains why the problems we discuss now did not receive full attention in their article.
}

\section{Using the Black Litterman Approach without an Equilibrium Model}

\subsection{How It Is Usually Done}

Jones, Lim, and Zangari (2007; henceafter JLZ) presented a way in which the Black Litterman model can be used to incorporate a factor-based view in a structured equity portfolio.

Let $\boldsymbol{w}$ be an $N$-dimensional portfolio weight vector, where the $i$-th element $w_{i}$ is the portfolio weight of asset $i$. Let $\boldsymbol{B}$ be an $N$-by- $K$ factor exposure matrix, where $(i, j)$ element $b_{i, j}$ is the exposure of stock $i$ to factor $j .^{4}$ Then the factor exposure of the portfolio is $\boldsymbol{B}^{\prime} \boldsymbol{w}$. A portfolio manager's factor view can be expressed as a $K$-dimensional vector $\lambda$, which is the portfolio manager's desired value of $\boldsymbol{B}^{\prime} \boldsymbol{w} .^{5}$

JLZ suggest the following steps for the portfolio construction:

1) Specify the factor view vector $\lambda$.

2) Calculate the optimal tilt portfolio weight, i.e. the weight of the portfolio that is optimal given the factor view vector. That is, solve:

$$
\min _{\boldsymbol{w}} \boldsymbol{w}^{\prime} \hat{\mathbf{\Sigma}} \boldsymbol{w} \quad \text { s.t. } \quad \boldsymbol{B}^{\prime} \boldsymbol{w}=\lambda
$$

where $\hat{\boldsymbol{\Sigma}}$ is an $N$-by- $N$ variance-covariance matrix estimate of asset returns. The solution to this problem is

$$
\boldsymbol{w}_{\text {ОTР }}=\hat{\boldsymbol{\Sigma}}^{-1} \boldsymbol{B}\left(\boldsymbol{B}^{\prime} \hat{\boldsymbol{\Sigma}}^{-1} \boldsymbol{B}\right)^{-1} \boldsymbol{\lambda}
$$

where OTP is an acronym for optimal tilt portfolio. ${ }^{6}$

3) Compute the Black Litterman alpha, i.e. the expected return that produces the optimal tilt portfolio identified in the previous step. That is, solve the following equation for $\alpha$ :

$$
\boldsymbol{w}_{\text {OTP }}=\operatorname{argmax} \quad \boldsymbol{w}^{\prime} \boldsymbol{\alpha}-\frac{1}{2} \boldsymbol{w}^{\prime} \hat{\boldsymbol{\Sigma}} \boldsymbol{w}
$$

The solution to this problem is ${ }^{7}$

$$
\boldsymbol{\alpha}_{\text {BL }}=\hat{\boldsymbol{\Sigma}} \boldsymbol{w}_{\text {OTP }}
$$

4) Find an optimal portfolio based on the Black Litterman alpha, $\boldsymbol{\alpha}_{B L}$, and any other constraints using a standard mean variance optimization. For example, an optimal portfolio can be found by solving the following quadratic programming problem:

$$
\begin{aligned}
& \max _{\boldsymbol{w}} \quad \boldsymbol{w}^{\prime} \boldsymbol{\alpha}_{B L}-\frac{1}{2} \boldsymbol{w}^{\prime} \hat{\boldsymbol{\Sigma}} \boldsymbol{w} \\
& \text { s.t. } \quad \boldsymbol{w}^{\prime} \boldsymbol{\imath}=1 \quad l_{i} \leq w_{i} \leq u_{i}, \quad i=1, \cdots, N
\end{aligned}
$$

\footnotetext{
${ }^{4}$ In many applications, $\boldsymbol{B}$ is a matrix of observable characteristics. ${ }^{5}$ Portfolio managers may determine the value of $\lambda$ based on their expectation of "factor returns." For example, if they expect the size of return to be high, they may choose a large value for the size exposure. ${ }^{6}$ The derivation of this result and other results is included in an appendix available at

http://papers.ssrn.com/sol3/papers.cfm?abstract_id $=2191278$.
} 
where $l_{i}$ and $u_{i}$ are lower and upper bounds on asset $i$ weights. $^{8}$

In the four step procedure described above, no explicit reference is made to an equilibrium model, which is an integral component of the original Black-Litterman model. In fact, there is no explicit reference to any model of stock returns.

Implicitly, though, the above procedure requires $a$ model to produce an estimate $\hat{\boldsymbol{\Sigma}}$. Even when one decides to use a historical variance-covariance matrix for $\hat{\boldsymbol{\Sigma}}$, one is using a model. The implicit model is that the historical variance-covariance matrix is a good estimator of the true variance-covariance matrix.

Using a model to produce an estimate $\hat{\boldsymbol{\Sigma}}$ and using the estimate in calculating the Black Litterman alpha, $\boldsymbol{\alpha}_{B L}$, however, is not equivalent to "combining a subjective view with an equilibrium model" as is done in the original Black Litterman model. The problem with this approach is that the model is used only to produce an estimate of the variance-covariance matrix. This approach ignores the information in the data about the mean return. ${ }^{9}$ The consequence is that a subjective view is combined with a model, but only partially and incompletely. The incompleteness does create a problem, as will be shown in the next sub-section.

\subsection{The Cost of Ignoring Mean Estimates}

The JLZ procedure calculates the Black Litterman alpha, $\boldsymbol{\alpha}_{B L}$, based on a view and a model. The view is summarized in $\lambda$ and the model is summarized in an estimate of $\boldsymbol{\Sigma}$. As mentioned above, however, the view and the model are not combined in an efficient way. In particular, the mean component of the model is ignored. This creates some loss, the magnitude of which we will quantify in this subsection.

We first discuss our measures of loss. In order to measure an investor's loss, we need to develop a formal model of returns and investor views. In the subsequent text, we discuss a measure of loss based upon a formal model of returns and views.

\subsubsection{A Measure of Loss}

One can quantify the loss generated in a portfolio construction process in terms of the Sharpe ratio, the information ratio, or an investor's utility. For the purpose at

\footnotetext{
${ }^{8}$ Without the constraints, the solution to this optimization would be the optimal tilt weights, $\boldsymbol{w}_{\text {OTP }}$.

${ }^{9}$ One might argue that means are typically not estimated accurately, and that ignoring the mean estimate is not a big deal. However, a variance estimate does not have the same meaning if one ignores the mean estimate. Recall that the variance measures the deviation from the mean. Without specifying a mean, a variance is an incomplete concept. One might end up making a decision as if the true mean is zero, which can be worse than using an imprecisely estimated mean.
}

hand, the most natural choice is to use the investor's utility implicit in the Black Litterman approach, which is

$$
U(\mu, \sigma)=\mu-\frac{\delta}{2} \sigma^{2}
$$

where $\mu$ is the mean return of the portfolio and $\sigma$ is the standard deviation of the portfolio return. In particular, we will set the value of $\delta$ to 1 , as was done in the JLZ procedure. ${ }^{10}$ Given the utility function, we can calculate the highest utility one can achieve given the model and the view, and we can also calculate the utility that one actually achieves. The difference is our measure of the loss.

To compute the utility, we will use the predictive mean and variance based on the model and the view. This is justified as our primary interest is in the investor's portfolio construction strategy, not the validity of the investor's belief. In addition to this, the predictive mean and variance are the only mean and variance that the investor can actually calculate. It would not make sense to define the investor's utility in terms of the quantities that are not known to the investor. ${ }^{11}$

If a portfolio construction process combines the model and the view efficiently, then by construction the loss is zero. This is true in the original Black Litterman approach. It is not possible to improve the utility given the model and the view. In the JLZ procedure, however, it is possible to improve the utility given the model and the view. It is possible to do so by adding the mean component of the model, as we will show below.

\subsubsection{The Model and the View}

Consider the following model:

$$
\boldsymbol{r}_{t+1}=\boldsymbol{B}_{t} \boldsymbol{f}+\boldsymbol{e}_{t+1}, \quad \boldsymbol{e}_{t+1} \sim N\left(0, \frac{1}{h} \boldsymbol{V}_{e}\right)
$$

where $\boldsymbol{r}_{t+1}$ represents the vector of stock returns, $\boldsymbol{B}_{t}$ represents the $N \times K$ matrix of factor exposures for $K$ factors and $N$ stocks, and $f$ is a $K \times 1$ vector of factor premiums, $\boldsymbol{e}_{t+1}$ is the error vector, and $\frac{1}{h} \boldsymbol{V}_{e}$ is the covariance matrix of the error. We include a scalar $h$ that affects the overall volatility of the market.

While this model is not explicitly stated in the four step procedure outlined in the previous section, this is probably the most natural model for the JLZ investor, if he were forced to adopt one. (Recall that the lack of an explicit model is one weakness of the JLZ procedure.) Without a model of this kind, it would be hard to mo-

\footnotetext{
${ }^{10}$ Our main arguments are not affected by the value of $\delta$, nor by the choice of how loss is measured.

${ }^{11}$ See the discussion preceeding Equation (14) for an example of alternatives that we did not take.
} 
tivate a factor tilted portfolio using the JLZ procedure. ${ }^{12}$

The variables $\boldsymbol{r}_{t+1}$ and $\boldsymbol{B}_{t}$ are observable, while $\boldsymbol{f}$ and $\boldsymbol{e}_{t+1}$ are not observable. The error variance $\frac{1}{h} \boldsymbol{V}_{e}$ is not known either. Before creating a portfolio for $\boldsymbol{T}+1$, the investor observes $\left(\boldsymbol{r}_{1}, \cdots, \boldsymbol{r}_{T}\right)$ and $\left(\boldsymbol{B}_{0}, \cdots, \boldsymbol{B}_{T-1}\right)$ and also $\boldsymbol{B}_{T}$. With these data, the investor must determine the distribution of $\boldsymbol{r}_{T+1}$, by estimating $\boldsymbol{f}, \boldsymbol{h}$, and $\boldsymbol{V}_{e}$, or by updating his belief about these quantities.

The JLZ procedure introduced $\lambda$ to capture the investor's belief. $\lambda$ is the desired tilt to specific factors, which depends on the investor's belief about factor returns. Rather than consider the prior of (i.e. distribution of possible values of) $\lambda$, we consider the prior of factor returns. While this approach may seem like an unnecessary detour, it makes the analysis much more tractable.

We interpret $\lambda$ in the context of the following prior of $\boldsymbol{f}$ and $h$ :

$$
\begin{aligned}
& \boldsymbol{f} \mid h \sim N\left(\boldsymbol{m}_{f}, \frac{1}{h} \boldsymbol{V}_{f}\right) \\
& h \sim G(p, q)
\end{aligned}
$$

That is, $\boldsymbol{f}$ has a multivariate normal distribution conditional on $h$, and $h$ has a Gamma distribution. ${ }^{13}$ By including $\frac{1}{h}$ in the variance of $f$, we are assuming that the overall uncertainty of the factor premium is proportional to the overall market volatility. ${ }^{14}$ This is the simplest prior one can adopt, and is general enough for our purpose. Satchell and Scowcroft (2000) also use this prior when they interpret the Black Litterman model. Note also that this prior allows for the noninformative prior as a special case, as will be shown in the next section.

It is easy to show that $\lambda$ is related to the parameters in Equations (7) and (8) in the following way:

$$
\lambda=\boldsymbol{B}^{\prime} \boldsymbol{\Sigma}^{-1} \boldsymbol{B} \boldsymbol{m}_{f}
$$

${ }^{12} \mathrm{JLZ}$ do mention this model and even suggest to use it to determine the view. We will discuss the problem of using a data based view in the next section. For now, we interpret the view as completely independent from the data.

${ }^{13}$ The Gamma distribution makes the analysis tractable. The Gamma distribution, when used with normal distribution, has the property that the posterior will be of the same type of distribution as the prior. Due to this property, the Gamma-Normal prior is called a conjugate prior, and is widely used in Bayesian analysis. We follow the parameterization of the Gamma distribution used by Koop (2003). The pdf of the above distribution is

$$
\left(\frac{2 p}{q}\right)^{-\frac{q}{2}} \Gamma\left(\frac{q}{2}\right)^{-1} h^{\frac{q}{2}-1} e^{-\frac{h q}{2 p}}
$$

${ }^{14}$ This aspect of the prior is required for a conjugate prior, i.e. a prior that leads to a posterior of the same distribution. Using a conjugate prior makes our analysis simple, but the conclusion of our analysis does not depend on a conjugate prior. where $\boldsymbol{\Sigma}$ is the variance-covariance matrix of $\boldsymbol{r}_{T+1}$, i.e., $\boldsymbol{B}_{T} \frac{1}{h} \boldsymbol{V}_{f} \boldsymbol{B}_{T}^{\prime}+E\left(\frac{1}{h} \boldsymbol{V}_{e}\right) \cdot{ }^{15}$

Equation (7) represents the model, and Equation (8) represents the investor's view. And together, they describe the belief system of the JLZ investor completely.

Let us be clear about our distinction between the model and the view. The model specifies the relationship among variables that we observe. The view is about the quantities that we do not observe. In particular, the view specifies the relationship among parameters of the model. The view has hyper-parameters, whose values investors choose on their own. In Equation (7), there are three parameters: $\boldsymbol{f}, \boldsymbol{V}_{e}, h$. In Equation (8), there are four hyper-parameters: $\boldsymbol{m}_{f}, \boldsymbol{V}_{f}, p, q$. The distinction between the parameters and the hyper-parameters can be made clearly: parameters are a part of the model, while hyper-parameters are a part of the view.

These concepts might be clearer if we describe them in a series of steps.

1) All the equations are known to everyone;

2) At the beginning of our portfolio formulation stage, God decides the value of the hyper-parameters

$\boldsymbol{m}_{f}, \boldsymbol{V}_{f}, p, q$. God announces this decision to every investor;

3) On day 1, God draws parameters $\boldsymbol{f}, \boldsymbol{V}_{e}, h$ using Equation (8). These quantities are never known to the investor;

4) On day 2 , God draws data $\left(\boldsymbol{r}_{1}, \cdots, \boldsymbol{r}_{T}\right)$, $\left(\boldsymbol{B}_{0}, \cdots, \boldsymbol{B}_{T-1}\right)$, and also $\boldsymbol{B}_{T}$, and shows them to the investor; ${ }^{16}$

5) After observing data, the investor estimates the

${ }^{15} \lambda$ is the factor tilt implied by the investor's view and the model. Thus, we can find an optimal portfolio given the investor's view and the model, and determine the factor tilt of that portfolio, which should be $\lambda$. Given the investor's view and the model, the unconditional distribution of $\boldsymbol{r}_{T+1}$ is

$$
\boldsymbol{r}_{T+1} \sim N\left(\boldsymbol{B}_{T} \boldsymbol{m}_{f}, \boldsymbol{\Sigma}\right)
$$

where $\boldsymbol{\Sigma}$ is the variance-covariance matrix of $\boldsymbol{r}_{T+1}$, i.e., $\boldsymbol{B}_{T} \frac{1}{h} \boldsymbol{V}_{f} \boldsymbol{B}_{T}^{\prime}+E\left(\frac{1}{h} \boldsymbol{V}_{e}\right)$. If the investor has the utility function specified in Equation (6) with $\delta$ equal to 1, then the optimal portfolio, given the above distribution, can be found from the following optimization:

$$
\max _{w} \boldsymbol{w}^{\prime} \boldsymbol{B}_{T} \boldsymbol{m}_{f}-\frac{1}{2}\left(\boldsymbol{w}^{\prime} \boldsymbol{\Sigma} \boldsymbol{w}\right)
$$

The solution to the above problem is

$$
\boldsymbol{w}^{*}=\boldsymbol{\Sigma}^{-1} \boldsymbol{B}_{T} \boldsymbol{m}_{f}
$$

From the factor tilt of this portfolio, we can obtain Equation (9). This comes from the second equation in the maximization problem, that $\boldsymbol{B}^{\prime} \boldsymbol{w}-\boldsymbol{\lambda}=0$. Thus, substitution of Equation 12 gives

$\lambda=\boldsymbol{B}^{\prime} \boldsymbol{w}=\boldsymbol{B}^{\prime} \boldsymbol{\Sigma}^{-1} \boldsymbol{B}_{T} \boldsymbol{m}_{f}$.

${ }^{16}$ Note that, in our analysis, $\boldsymbol{B}$ 's are matrices of observable characteristics, not estimates of factor loadings. Estimation error does not arise as far as $\boldsymbol{B}$ 's are concerned. 
parameters, makes a prediction of $\boldsymbol{r}_{T+1}$, and creates a portfolio;

6) On day 3, God draws $\boldsymbol{r}_{T+1}$ using Equation (7);

7) After observing $\boldsymbol{r}_{T+1}$, the investor calculates his portfolio return.

In this scheme, one could think of a number of different expectations and variances of the portfolio return. Let us consider three of them. First, we can think of "God's expectation," which is conditional on the true value of parameters $\boldsymbol{f}, h$, and $\boldsymbol{V}_{e}$. Since only God knows the value of $\boldsymbol{f}, h$, and $\boldsymbol{V}_{e}$, this expectation is probably not very interesting to our hypothetical investor. Second, we can think of the "predictive expectation," which is conditional on the data as well as on the hyperparameters, but not on the true value of the parameters. That is, it is the expectation based on the investor's best prediction given the information available. This is something rational investors can calculate. Third, we can think of "an economist's expectation," which is conditional only on the hyper-parameters $\boldsymbol{m}_{f}, \boldsymbol{V}_{f}, p, q$. This is the expectation one obtains by imagining many different worlds where different parameters could have been chosen from the same hyper-parameters. To investors who live only once, this concept is not particularly interesting. Their utility does not depend on what could have happened in other worlds. This concept, however, could be interesting to economists who want to make general statements about certain decision making schemes. Note also that "an economist's expectation" is obtained if we integrate the data out of the "predictive expectation."17

Our analysis is based on the "predictive expectation" and similar concepts, as we are working with a utilitybased measure of loss. Predictive expectation is conditional on the value of the hyper-parameters. Thus, an analysis of predictive expectation can be influenced by the choice of hyper-parameter values. In our numerical analysis, we vary the values of the hyper-parameters to make sure that our results are robust, and that they are not peculiar to the chosen values of the hyper-parameters.

We provide the formula for the predictive mean and the predictive variance-covariance matrix of the returns here without proof. ${ }^{18}$ The predictive mean and the predictive variance-covariance matrix of returns are obtained by applying statistical operators to the model and then

\footnotetext{
${ }^{17}$ That is,

$E$ (portfolioreturn|hyperparameters)

$=\int E($ portfolioreturn $\mid$ hyperparameters, data $) p($ data $)$

where $p($ data $)$ is the probability density function of the data. The integration is taken over the data. In other words, if we average the predictive expectation over all the possible values of the data, then we get an economist's expectation.

${ }^{18}$ The derivation of this result is included in an appendix available at http://papers.ssrn.com/sol3/papers.cfm?abstract_id=2191278.
}

using the Bayesian updating formula (see Zellner [7] and Koop [8]). Let us denote the predictive mean of the return as $\tilde{E}\left(\boldsymbol{r}_{T+1}\right)$ and the predictive variance-covariance matrix of the return as $\tilde{V}\left(\boldsymbol{r}_{T+1}\right)$. Then

$$
\begin{aligned}
& \tilde{E}\left(\boldsymbol{r}_{T+1}\right)=\boldsymbol{B}_{T} \tilde{E}(\boldsymbol{f}) \\
& \tilde{V}\left(\boldsymbol{r}_{T+1}\right)=\boldsymbol{B}_{T} \tilde{V}(\boldsymbol{f}) \boldsymbol{B}_{T}^{\prime}+\tilde{V}\left(\boldsymbol{e}_{T+1}\right)
\end{aligned}
$$

The predictive mean of the factor premium $\tilde{E}(\boldsymbol{f})$, the predictive variance-covariance matrix of the factor premium $\tilde{V}(\boldsymbol{f})$, and the predictive variance-covariance matrix of the error $\tilde{V}\left(\boldsymbol{e}_{T+1}\right)$ are determined by the Bayesian updating formula:

$$
\begin{aligned}
& \tilde{E}(\boldsymbol{f})=\left(\boldsymbol{V}_{f}^{-1}+\boldsymbol{S}_{B}\right)^{-1}\left(\boldsymbol{V}_{f}^{-1} \boldsymbol{m}_{f}+\boldsymbol{S}_{B} \hat{\hat{\boldsymbol{f}}}\right) \\
& \tilde{V}(\boldsymbol{f})=\frac{q+N T}{q+N T-2} \frac{1}{\tilde{E}(h)}\left(\boldsymbol{V}_{f}^{-1}+\boldsymbol{S}_{B}\right)^{-1} \\
& \tilde{V}\left(\boldsymbol{e}_{T+1}\right)=\frac{1}{\tilde{E}(h)} \tilde{V}_{e}
\end{aligned}
$$

The first equation simply says that the predictive mean of the factor premium is a "precision-weighted" average of the prior mean $\boldsymbol{m}_{f}$ and the GLS estimator $\hat{\boldsymbol{f}}$. The second and the third equation simply break down the components of the variance-covariance matrix. The predictive mean of the error precision parameter $\tilde{E}(h)$ can be expressed as follows: ${ }^{19}$

$$
\begin{aligned}
& \frac{1}{\tilde{E}(h)} \\
& =\frac{1}{q+N T}\left[\frac{q}{p}+\boldsymbol{S}_{e}+\left(\boldsymbol{m}_{f}-\hat{\hat{\boldsymbol{f}}}\right)^{\prime}\left(\boldsymbol{V}_{f}+\boldsymbol{S}_{B}^{-1}\right)^{-1}\left(\boldsymbol{m}_{f}-\hat{\hat{\boldsymbol{f}}}\right)\right]
\end{aligned}
$$

\subsubsection{An Analytic Formula for Loss}

Now we are ready to compare the utility of an investor who follows the JLZ procedure (the JLZ investor) to the utility of a rational investor who combines all information efficiently (the RTL investor), both having the same view and the same model. We first discuss the utility of the two investors in general form. Then we examine a special case where we can provide an analytic solution for loss.

Let us first characterize the utility of the RTL investor. As noted before, the investor would not consider $\boldsymbol{B} \boldsymbol{m}_{f}$

$$
\begin{aligned}
& { }^{19} \text { The GLS estimator of the factor premium } \hat{\hat{\boldsymbol{f}}} \text { is } \\
& \left(\sum_{t=0}^{T-1} \boldsymbol{B}_{t}^{\prime} \hat{\boldsymbol{V}}_{e}^{-1} \boldsymbol{B}_{t}\right)^{-1} \sum_{t=0}^{T-1} \boldsymbol{B}_{t}^{\prime} \hat{\boldsymbol{V}}_{e}^{-1} \boldsymbol{r}_{t+1} \text {, its precision matrix } \boldsymbol{S}_{B} \text { is } \\
& \sum_{t=0}^{T-1} \boldsymbol{B}_{t}^{\prime} \hat{\boldsymbol{V}}_{e}^{-1} \boldsymbol{B}_{t} \text {, and the error sum of square matrix } \boldsymbol{S}_{e} \text { is } \\
& \sum_{t=0}^{T-1}\left(\boldsymbol{r}_{t+1}-\boldsymbol{B}_{t} \hat{\hat{\boldsymbol{f}}}\right)^{\prime} \hat{\boldsymbol{V}}_{e}^{-1}\left(\boldsymbol{r}_{t+1}-\boldsymbol{B}_{t} \hat{\hat{\boldsymbol{f}}}\right) \text {, where } \hat{\boldsymbol{f}} \text { is } \\
& \left(\sum_{t=0}^{T-1} \boldsymbol{B}_{t}^{\prime} \boldsymbol{B}_{t}\right)^{-1} \sum_{t=0}^{T-1} \boldsymbol{B}_{t}^{\prime} \boldsymbol{r}_{t+1} \text {, and } \hat{\boldsymbol{V}}_{e} \text { is } \frac{p}{T} \sum_{t=0}^{T-1}\left(\boldsymbol{r}_{t+1}-\boldsymbol{B}_{t} \hat{\boldsymbol{f}}\right)\left(\boldsymbol{r}_{t+1}-\boldsymbol{B}_{t} \hat{\boldsymbol{f}}\right)^{\prime} .
\end{aligned}
$$


to be the best estimate of the mean return, nor would he consider the Black Litterman alpha implied by the optimal tilted portfolio to be the best estimate of the mean return. Using the Black Litterman alpha would be equivalent to not using any information from the estimation about the mean return. Instead, the RTL investor would estimate the mean and the variance-covariance matrix of returns using all the information available, and then choose a portfolio based on these estimates.

The best estimates of the mean and of the variancecovariance matrix of the returns are the predictive mean and the predictive variance-covariance matrix described above. Given the predictive mean and the predictive variance-covariance matrix of the return, the rational investor will choose the portfolio by solving the optimization problem comparable to Equation (5). Then his utility is determined as: ${ }^{20}$

$$
U_{R T L}=\boldsymbol{w}_{R T L} \tilde{E}\left(\boldsymbol{r}_{T+1}\right)-\frac{1}{2} \boldsymbol{w}_{R T L} \tilde{V}\left(\boldsymbol{r}_{T+1}\right) \boldsymbol{w}_{R T L}
$$

where

$$
\begin{aligned}
& \boldsymbol{w}_{R T L}=\operatorname{argmax}_{w} \quad \boldsymbol{w}^{\prime} \tilde{E}\left(\boldsymbol{r}_{T+1}\right)-\frac{1}{2} \boldsymbol{w}^{\prime} \tilde{V}\left(\boldsymbol{r}_{T+1}\right) \boldsymbol{w} \\
& \text { s.t. } \quad \boldsymbol{w}^{\prime} \boldsymbol{\imath}=1, \quad l_{i} \leq w_{i} \leq u_{i}, \quad i=1, \cdots, N
\end{aligned}
$$

Now let us consider the utility of the JLZ investor. The JLZ investor would follow the four steps specified in the previous section. Thus, her portfolio is the solution to Equation (5), and her utility is determined from the mean and the variance of her portfolio. That is,

$$
U_{J L Z}=\boldsymbol{w}_{J L Z} \tilde{E}\left(\boldsymbol{r}_{T+1}\right)-\frac{1}{2} \boldsymbol{w}_{J L Z} \tilde{V}\left(\boldsymbol{r}_{T+1}\right) \boldsymbol{w}_{J L Z}
$$

where

$$
\begin{aligned}
& \boldsymbol{w}_{J L Z}=\operatorname{argmax}_{\boldsymbol{w}} \\
& \boldsymbol{w}^{\prime} \boldsymbol{B}_{T}\left(\boldsymbol{B}_{T} \tilde{V}\left(\boldsymbol{r}_{T+1}\right)^{-1} \boldsymbol{B}_{T}\right)^{-1} \lambda-\frac{1}{2} \boldsymbol{w}^{\prime} \tilde{V}\left(\boldsymbol{r}_{T+1}\right) \boldsymbol{w} \\
& \text { s.t. } \quad \boldsymbol{w}^{\prime} \boldsymbol{l}=1, \quad l_{i} \leq w_{i} \leq u_{i}, \quad i=1, \cdots, N
\end{aligned}
$$

Computation of the loss based on the above utility can be done numerically, which we explain in the next subsection. For now, we will present an analytic solution for the special case where there are no constraints in the optimization. Although, this is not a realistic way of constructing an optimal portfolio, it will help illustrate some key concepts.

Let us start with the rational investor. When there are no constraints, the solution to the optimization problem in Equation (18) is simply

$$
\boldsymbol{w}_{R T L}=\tilde{V}\left(\boldsymbol{r}_{T+1}\right)^{-1} \tilde{E}\left(\boldsymbol{r}_{T+1}\right)
$$

\footnotetext{
${ }^{20}$ Recall that we evaluate the utility in terms of predictive mean and variance.
}

Then the utility is given by

$$
U_{R T L}=\frac{1}{2} \tilde{E}\left(\boldsymbol{r}_{T+1}^{\prime}\right) \tilde{V}\left(\boldsymbol{r}_{T+1}\right)^{-1} \tilde{E}\left(\boldsymbol{r}_{T+1}\right)
$$

Now let us calculate the utility of the JLZ investor. When there are no constraints, the portfolio of the JLZ investor is simply the optimal tilted portfolio of Equation (2). Combining Equation (2) and Equation (9), we get

$$
\begin{aligned}
& \boldsymbol{w}_{J L Z}=\tilde{V}\left(\boldsymbol{r}_{T+1}\right)^{-1} \boldsymbol{B}_{T+1} \boldsymbol{m}_{f} \\
& =\boldsymbol{w}_{R T L}+\tilde{V}\left(\boldsymbol{r}_{T+1}\right)^{-1} \boldsymbol{B}_{T+1}\left(\boldsymbol{m}_{f}-\tilde{E}(\boldsymbol{f})\right)
\end{aligned}
$$

Note that we made one simplifying assumption to get the above formula. Firstly, we assumed that the JLZ investor's covariance estimate of returns $(\hat{\boldsymbol{\Sigma}})$ is identical to the rational investor's covariance estimate of returns $\left(\tilde{V}\left(\boldsymbol{r}_{T+1}\right)\right)$. Since we did not specify how the JLZ investor obtains $\hat{\boldsymbol{\Sigma}}$, we chose the one that was the best and most advantageous to the JLZ investor. In other words, by doing this we eliminated one mistake that the JLZ investor made, i.e. using an inferior estimate of the error covariance.

Now we can express the utility of the JLZ investor in the following way:

$$
\begin{aligned}
& U_{J L Z}=\boldsymbol{w}_{J L Z}^{\prime} \tilde{E}\left(\boldsymbol{r}_{T+1}\right)-\frac{1}{2} \boldsymbol{w}_{J L Z}^{\prime} \tilde{V}\left(\boldsymbol{r}_{T+1}\right) \boldsymbol{w}_{J L Z} \\
& =U_{R T L}-\frac{1}{2}\left[\boldsymbol{m}_{f}-\tilde{E}(\boldsymbol{f})\right]^{\prime} \boldsymbol{B}_{T+1} \tilde{V}\left(\boldsymbol{r}_{T+1}\right)^{-1} \\
& \cdot \boldsymbol{B}_{T+1}\left[\boldsymbol{m}_{f}-\tilde{E}(\boldsymbol{f})\right]
\end{aligned}
$$

The above formula shows that the difference in utility between the JLZ investor and the RTL investor, i.e. the loss, is quadratic in the difference between the prior mean and the posterior mean of the factor premium. This can be understood intuitively. When one ignores the mean estimates, the portfolio weights deviate from the optimal weights and the deviation is linear in the difference between the prior mean and the posterior mean. The utility, however, depends on the second moments of the portfolio returns, so the loss is quadratic in the difference between the prior mean and the posterior mean.

\subsubsection{Investor Simulations}

In order to quantify the losses from an incorrect procedure, we perform a numerical simulation.

The simulation is based on the monthly returns and other characteristics of the MSCI country indices. ${ }^{21}$ Specifically, we select the top 10 countries by market capitalization. We used the price-to-earnings ratio, 6 months momentum return, and the market capitalization of the indices as factors in our factor model. Our data set

\footnotetext{
${ }^{21}$ We could have carried out the simulations at the individual stock level. All our formulas would work at the individual stock level as well.
} 
spans the 7-year period from September 2001 to August 2008. The summary statistics of the variables for each country are presented in Table 1.

For the simulation, we use the Chi-square distribution for the error precision parameter $h$. The Chi-square distribution is a special case of the Gamma distribution, and all the formulas presented above are still applicable. The model and the prior now look as follows:

$$
\boldsymbol{r}_{t+1}=\boldsymbol{B}_{t} \boldsymbol{f}+\boldsymbol{e}_{t+1}, \quad \boldsymbol{e}_{t+1} \sim N\left(0, \frac{1}{h} \boldsymbol{V}_{e}\right)
$$

and

$$
\begin{aligned}
& \boldsymbol{f} \mid h \sim N\left(\boldsymbol{m}_{f}, \frac{1}{h} \boldsymbol{V}_{f}\right) \\
& h \sim \chi(p)
\end{aligned}
$$

The simulation proceeds according to the following steps. $^{22}$

1) We choose a reasonable value for the hyper parameters $\boldsymbol{m}_{f}, \boldsymbol{V}_{f}, p$;

2) We draw a single value of parameters $f$ and $h$ using the hyper parameters above. We use the OLS estimator for the remaining parameter $\boldsymbol{V}_{e}$;

3) We draw $\left(\boldsymbol{r}_{1}, \cdots, \boldsymbol{r}_{T}\right)$ from the parameters determined above. For the factor exposures $\left(\boldsymbol{B}_{0}, \cdots, \boldsymbol{B}_{T-1}\right)$ and $\boldsymbol{B}_{T}$, we use historical values;

4) Given the values above, we determine the portfolio of the JLZ investor and the RTL investor;

5) We calculate the loss;

6) We repeat Steps 2 to 5 a 1000 times, without changing the hyper parameters. Then we obtain a distribution of losses, which will allow us to make general statements about the JLZ investor's decision making process;

7) We repeat the above six steps by making small perturbations to the hyper parameter values.

The outcome of the simulations is summarized in Tables 2 and 3. A figure of the actual losses in 1000 simulations is shown in Figure 1. The magnitude of the loss is fairly stable across various sets of hyper parameter values. The mean loss is between 0.10 and 0.15 , while the median loss is between 0.06 and 0.11 . As the loss is the difference in the quadratic utility, we could interpret the loss as a reduction in the expected monthly return. That is, the loss of the JLZ investor amounts to about a $0.1 \%$ reduction in the expected monthly return. This would imply a fairly conservative estimate of the return loss as $1.2 \%$ annually. $^{23}$

\footnotetext{
${ }^{22}$ Further details about the simulation procedure are provided in an appendix available at

http://papers.ssrn.com/sol3/papers.cfm?abstract_id=2191278.

${ }^{23}$ This estimate is fairly conservative, as we allow the JLZ investor to use the "correct" variance-covariance matrix of errors.
}

\begin{tabular}{|c|c|c|c|c|c|c|}
\hline Country & Variable & Nobs & Mean & SD & Min & Max \\
\hline \multirow[t]{4}{*}{ Australia } & Return & 84 & 1.50 & 4.95 & -12.57 & 14.84 \\
\hline & Market Cap & 84 & 5.04 & 2.27 & 2.02 & 10.13 \\
\hline & $\mathrm{P} / \mathrm{E}$ & 84 & 18.01 & 2.17 & 13.51 & 24.02 \\
\hline & Momentum & 84 & 11.39 & 11.46 & -14.33 & 33.07 \\
\hline \multirow[t]{4}{*}{ Canada } & Return & 84 & 1.46 & 4.88 & -11.26 & 9.78 \\
\hline & Market Cap & 84 & 6.74 & 3.16 & 2.69 & 12.68 \\
\hline & $\mathrm{P} / \mathrm{E}$ & 84 & 19.95 & 4.04 & 16.09 & 32.53 \\
\hline & Momentum & 84 & 10.12 & 12.76 & -19.41 & 33.30 \\
\hline \multirow[t]{4}{*}{ France } & Return & 84 & 0.83 & 5.11 & -15.34 & 15.63 \\
\hline & Market Cap & 84 & 9.21 & 3.25 & 4.28 & 15.05 \\
\hline & $\mathrm{P} / \mathrm{E}$ & 84 & 23.28 & 13.66 & 10.70 & 70.26 \\
\hline & Momentum & 84 & 5.96 & 13.64 & -29.15 & 33.91 \\
\hline \multirow[t]{4}{*}{ Germany } & Return & 84 & 1.06 & 6.66 & -24.35 & 23.42 \\
\hline & Market Cap & 84 & 6.93 & 2.94 & 2.69 & 13.09 \\
\hline & $\mathrm{P} / \mathrm{E}$ & 84 & 19.50 & 27.84 & -211.30 & 68.96 \\
\hline & Momentum & 84 & 7.45 & 18.67 & -39.44 & 50.42 \\
\hline \multirow[t]{4}{*}{ Italy } & Return & 84 & 0.76 & 5.00 & -13.78 & 13.62 \\
\hline & Market Cap & 84 & 3.60 & 1.12 & 1.78 & 5.52 \\
\hline & $\mathrm{P} / \mathrm{E}$ & 84 & 17.92 & 4.89 & 9.83 & 38.65 \\
\hline & Momentum & 84 & 5.87 & 13.16 & -26.41 & 28.19 \\
\hline \multirow[t]{4}{*}{ Japan } & Return & 84 & 0.46 & 4.62 & -9.34 & 13.43 \\
\hline & Market Cap & 84 & 20.50 & 6.70 & 10.19 & 30.31 \\
\hline & $\mathrm{P} / \mathrm{E}$ & 84 & 15.09 & 55.45 & -175.67 & 109.71 \\
\hline & Momentum & 84 & 3.03 & 16.01 & -32.99 & 36.54 \\
\hline \multirow[t]{4}{*}{ Spain } & Return & 84 & 1.31 & 5.57 & -16.19 & 14.79 \\
\hline & Market Cap & 84 & 3.56 & 1.43 & 1.44 & 6.18 \\
\hline & $\mathrm{P} / \mathrm{E}$ & 84 & 15.28 & 1.83 & 9.90 & 18.79 \\
\hline & Momentum & 84 & 9.69 & 14.24 & -26.17 & 36.12 \\
\hline \multirow[t]{4}{*}{ Switzerland } & Return & 84 & 0.85 & 3.76 & -12.02 & 11.35 \\
\hline & Market Cap & 84 & 6.55 & 1.84 & 3.88 & 9.55 \\
\hline & $\mathrm{P} / \mathrm{E}$ & 84 & 20.33 & 4.49 & 12.92 & 32.88 \\
\hline & Momentum & 84 & 6.04 & 11.30 & -19.37 & 26.98 \\
\hline \multirow[t]{4}{*}{ UK } & Return & 84 & 0.69 & 3.88 & -10.35 & 10.40 \\
\hline & Market Cap & 84 & 22.42 & 6.00 & 13.29 & 33.61 \\
\hline & $\mathrm{P} / \mathrm{E}$ & 84 & 14.47 & 2.23 & 9.88 & 19.71 \\
\hline & Momentum & 84 & 5.22 & 11.59 & -19.04 & 27.67 \\
\hline \multirow[t]{4}{*}{ USA } & Return & 84 & 0.33 & 3.71 & -11.33 & 9.04 \\
\hline & Market Cap & 84 & 105.25 & 21.13 & 66.22 & 140.48 \\
\hline & $\mathrm{P} / \mathrm{E}$ & 84 & 20.79 & 4.77 & 15.69 & 34.60 \\
\hline & Momentum & 84 & 1.98 & 10.49 & -29.37 & 22.93 \\
\hline
\end{tabular}

Table 1. Summary Statistics of Returns and Factor Exposures.

Note: Return is the monthly percentage return. Market cap is the market capitalization in hundred of billions of US dollars. P/E is the price-toearnings ratio as calculated by MSCI. Momentum is latest 6 month return. 
Table 2. Utility of the JLZ, FFK, and Rational Investor from Various Values of $\boldsymbol{m}_{f}$ and $\boldsymbol{p}$.

\begin{tabular}{|c|c|c|c|c|c|}
\hline H.P. Set & Mean & Median & S.D. & Max & Min \\
\hline \multirow[t]{3}{*}{1.00} & -0.99 & -0.79 & 1.32 & 1.64 & -10.58 \\
\hline & -1.10 & -0.92 & 1.27 & 1.44 & -10.63 \\
\hline & -1.09 & -0.89 & 1.30 & 1.43 & -11.97 \\
\hline \multirow[t]{3}{*}{2.00} & -0.78 & -0.53 & 1.39 & 1.83 & -9.05 \\
\hline & -0.90 & -0.63 & 1.35 & 1.57 & -9.05 \\
\hline & -0.89 & -0.60 & 1.38 & 1.57 & -10.15 \\
\hline \multirow[t]{3}{*}{3.00} & -1.15 & -0.89 & 1.42 & 1.48 & -10.73 \\
\hline & -1.28 & -1.00 & 1.38 & 1.22 & -10.79 \\
\hline & -1.26 & -0.98 & 1.40 & 1.46 & -11.40 \\
\hline \multirow[t]{3}{*}{4.00} & -1.11 & -0.85 & 1.43 & 1.71 & -11.59 \\
\hline & -1.23 & -0.97 & 1.38 & 1.29 & -11.62 \\
\hline & -1.23 & -0.97 & 1.40 & 1.27 & -11.61 \\
\hline \multirow[t]{3}{*}{5.00} & -1.09 & -0.79 & 1.57 & 1.55 & -15.13 \\
\hline & -1.19 & -0.90 & 1.53 & 1.28 & -15.14 \\
\hline & -1.18 & -0.87 & 1.57 & 1.29 & -15.97 \\
\hline \multirow[t]{3}{*}{6.00} & -0.94 & -0.66 & 1.41 & 2.30 & -15.90 \\
\hline & -1.07 & -0.80 & 1.37 & 2.03 & -15.92 \\
\hline & -1.05 & -0.77 & 1.39 & 2.02 & -16.14 \\
\hline \multirow[t]{3}{*}{7.00} & -0.78 & -0.51 & 1.33 & 1.84 & -9.68 \\
\hline & -0.90 & -0.64 & 1.29 & 1.62 & -9.70 \\
\hline & -0.89 & -0.60 & 1.31 & 1.52 & -10.42 \\
\hline \multirow[t]{3}{*}{8.00} & -1.27 & -1.02 & 1.46 & 2.21 & -8.94 \\
\hline & -1.38 & -1.09 & 1.42 & 1.81 & -8.94 \\
\hline & -1.37 & -1.08 & 1.46 & 1.82 & -10.18 \\
\hline \multirow[t]{3}{*}{9.00} & -0.97 & -0.69 & 1.41 & 1.95 & -13.60 \\
\hline & -1.08 & -0.82 & 1.37 & 1.45 & -13.60 \\
\hline & -1.07 & -0.80 & 1.38 & 1.57 & -13.93 \\
\hline \multirow[t]{3}{*}{10.00} & -1.01 & -0.73 & 1.31 & 1.82 & -8.61 \\
\hline & -1.12 & -0.81 & 1.28 & 1.46 & -8.61 \\
\hline & -1.11 & -0.79 & 1.30 & 1.50 & -8.61 \\
\hline \multirow[t]{3}{*}{11.00} & -0.71 & -0.46 & 1.39 & 1.72 & -10.85 \\
\hline & -0.86 & -0.57 & 1.33 & 1.40 & -10.89 \\
\hline & -0.83 & -0.56 & 1.35 & 1.40 & -11.08 \\
\hline
\end{tabular}

Note: The H.P. is for Hyper-Parameter set. Sets $1-11$ alter the value of

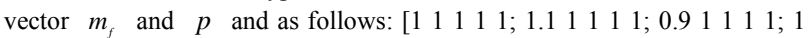
$1.1111 ; 10.9111 ; 111.111 ; 110.911 ; 1111.11 ; 1110.91 ; 1111$ $0.9 ; 1 \quad 1 \quad 1 \quad 11.1]$. Each set is separated by a semi-colon. For example, set 1 is the baseline case where $m_{f}$ is not altered. Set 2 alters $m_{f}$ by multiplying the 2nd element of $m_{f}$ by 1.1 and so on and so forth. Each row in the table within each set represents the utility for a particular investor. The first row is Rational, the second row is the JLZ, and the third row is the FFK investor.
Table 3. Utility Losses to the JLZ and FFK Investors from Various Values of $\boldsymbol{m}_{f}$ and $\boldsymbol{p}$.

\begin{tabular}{llllll}
\hline H.P. Set & Mean & Median & S.D. & Max & Min \\
\hline 1.00 & 0.11 & 0.08 & 0.12 & 1.21 & 0.00 \\
& 0.09 & 0.06 & 0.10 & 1.39 & 0.00 \\
2.00 & 0.12 & 0.08 & 0.13 & 1.24 & 0.00 \\
& 0.11 & 0.08 & 0.12 & 1.10 & 0.00 \\
3.00 & 0.13 & 0.09 & 0.14 & 1.37 & 0.00 \\
& 0.11 & 0.08 & 0.11 & 1.07 & 0.00 \\
4.00 & 0.13 & 0.09 & 0.12 & 0.84 & 0.00 \\
& 0.12 & 0.08 & 0.11 & 0.88 & 0.00 \\
5.00 & 0.10 & 0.06 & 0.12 & 0.82 & 0.00 \\
& 0.09 & 0.06 & 0.11 & 1.46 & 0.00 \\
6.00 & 0.13 & 0.09 & 0.13 & 1.14 & 0.00 \\
& 0.11 & 0.08 & 0.11 & 0.80 & 0.00 \\
7.00 & 0.12 & 0.09 & 0.13 & 1.00 & 0.00 \\
& 0.11 & 0.08 & 0.11 & 1.38 & 0.00 \\
8.00 & 0.12 & 0.07 & 0.14 & 1.19 & 0.00 \\
& 0.11 & 0.07 & 0.13 & 1.58 & -0.00 \\
\multirow{2}{*}{10.00} & 0.12 & 0.08 & 0.12 & 0.85 & 0.00 \\
& 0.10 & 0.07 & 0.10 & 1.30 & 0.00 \\
& 0.11 & 0.08 & 0.13 & 0.91 & 0.00 \\
& 0.10 & 0.07 & 0.11 & 1.03 & 0.00 \\
& 0.15 & 0.11 & 0.15 & 1.10 & 0.00 \\
& 0.12 & 0.10 & 0.11 & 0.79 & 0.00 \\
\hline
\end{tabular}

Note: The H.P. is for Hyper-Parameter set. Sets $1-11$ alter the value of

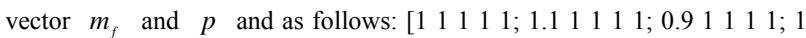
$1.1111 ; 10.9111 ; 111.111 ; 110.911 ; 1111.11 ; 1110.91 ; 1111$ $0.9 ; 1 \quad 1 \quad 1 \quad 11.1]$. Each set is separated by a semi-colon. For example, set 1 is the baseline case where $m_{f}$ is not altered. Set 2 alters $m_{f}$ by multiplying the 2nd element of $m_{f}$ by 1.1 and so on and so forth. The first row represents the utility loss to the JLZ investor and the second row represents the utility loss to the FFK investor for each hyper parameter set.

\section{Using the Black-Litterman Approach with a Data Based Prior}

\subsection{How It Is Usually Done}

Fabozzi, Forcardi, and Kolm (2006; henceafter FFK) suggested that one could incorporate a trading strategy, possibly based on a factor model, as a prior in the Black Litterman approach. The portfolio construction process might look as follows. ${ }^{24}$

1) Estimate the factor model that represents the portfolio manager's belief, and specify the "prior" from the estimates of the model. Suppose that the factor model can be written in the following form

\footnotetext{
${ }^{24}$ See p. 32 of Fabbozi, Forcardi, and Kolm (2006).
} 


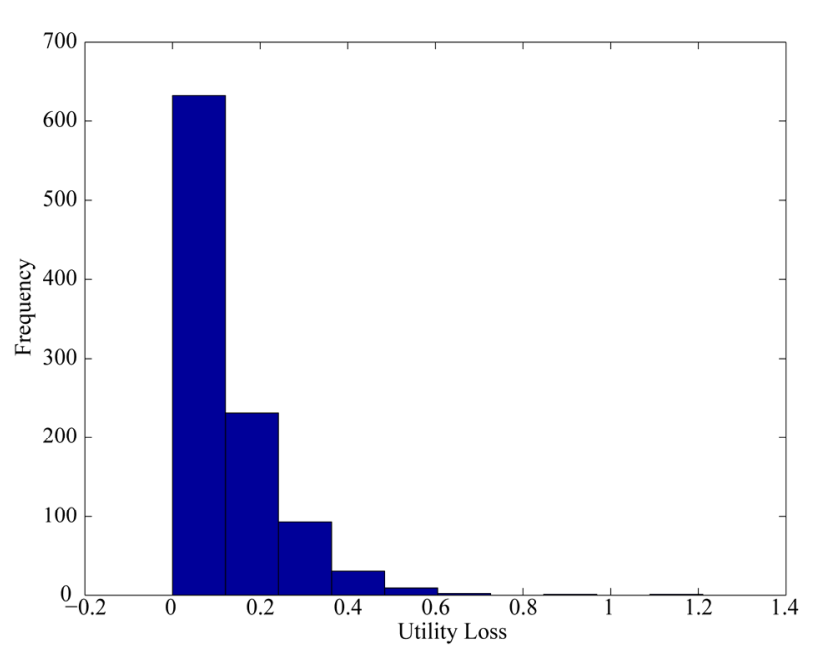

Figure 1. Utility Losses from 1000 Simulations for the JLZ Investor.

$$
\boldsymbol{r}_{t+1}=\boldsymbol{B}_{t} \boldsymbol{f}+\boldsymbol{e}_{t+1}, \quad \boldsymbol{e}_{t+1} \sim N\left(0, \boldsymbol{\Sigma}_{e}\right)
$$

Given the estimate $\hat{\boldsymbol{f}}$ and $\hat{\boldsymbol{\Sigma}}_{e}$, the "prior" for the mean of the future return, $\boldsymbol{g} \equiv E\left(\boldsymbol{r}_{T+1}\right)$, can be specified as: $:^{25}$

$$
\boldsymbol{g} \sim N\left(\boldsymbol{B}_{T} \hat{\boldsymbol{f}}, \hat{\boldsymbol{\Sigma}}_{e}\right)
$$

2) Let $\pi$ be the estimate of the "equilibrium return" implied by the Capital Asset Pricing Model. Assume the following "model" for $\boldsymbol{\pi}$ :

$$
\boldsymbol{\pi}=\boldsymbol{g}+\boldsymbol{\varepsilon}, \quad \boldsymbol{\varepsilon} \sim N(0, \tau \boldsymbol{\Sigma})
$$

where $\boldsymbol{\Sigma}$ is the variance-covariance matrix of future returns, i.e., $\boldsymbol{\Sigma} \equiv V\left(\boldsymbol{r}_{T+1}\right)$, and $\tau$ is a known constant. Note that $\boldsymbol{\pi}=\hat{\boldsymbol{\Sigma}} \boldsymbol{w}_{M}$, where $\hat{\boldsymbol{\Sigma}}$ is an estimate of $\boldsymbol{\Sigma} .^{26}$ From this value of $\boldsymbol{\pi}$, one can get an estimate of $\boldsymbol{g}$ and also get a variance-covariance matrix estimate of the estimator:

$$
\hat{\boldsymbol{g}} \equiv \boldsymbol{\pi}, \quad \hat{\boldsymbol{V}}(\hat{\boldsymbol{g}}) \equiv \tau \hat{\boldsymbol{\Sigma}}
$$

3) Combine the "prior" with the likelihood of the "model" according to Bayes rule. The posterior of the mean returns $\boldsymbol{g}$ is specified by ${ }^{27}$

\footnotetext{
${ }^{25} \hat{\Sigma}_{e}$ is an estimate of the error variance, not an estimate of the uncertainty of the mean estimate. In some special cases, however, $\hat{\boldsymbol{\Sigma}}_{e}$ can be interpreted as as estimate of the uncertainty of the mean estimate, for example, if there is only one variable on the right hand side of the model.

${ }^{26}$ See the section entitled "The Derivation of the CAPM Equilibrium Return $\pi$ " in the appendix available at

http://papers.ssrn.com/sol3/papers.cfm?abstract_id=2191278 for a detailed explanation.

${ }^{27}$ See the appendix available at

http://papers.ssrn.com/sol3/papers.cfm?abstract_id=2191278 for the proof.
}

$$
\begin{aligned}
& \hat{E}(\boldsymbol{g}) \equiv\left(\hat{\boldsymbol{\Sigma}}_{e}^{-1}+\frac{1}{\tau} \hat{\boldsymbol{\Sigma}}^{-1}\right)^{-1}\left(\hat{\boldsymbol{\Sigma}}_{e}^{-1} \boldsymbol{B}_{T} \hat{\boldsymbol{f}}+\frac{1}{\tau} \hat{\boldsymbol{\Sigma}}^{-1} \boldsymbol{\pi}\right) \\
& \hat{V}(\boldsymbol{g}) \equiv\left(\hat{\boldsymbol{\Sigma}}_{e}^{-1}+\frac{1}{\tau} \hat{\boldsymbol{\Sigma}}^{-1}\right)^{-1}
\end{aligned}
$$

The posterior mean $\hat{E}(\boldsymbol{g})$ is sometimes referred to as the "Black Litterman alpha".

4) Find the optimal portfolio from a mean variance optimization using the posterior mean $\hat{E}(\boldsymbol{g})$ and the posterior variance-covariance matrix of stock returns $\hat{\boldsymbol{\Sigma}} .^{28}$ That is, solve

$$
\begin{array}{ll}
\max _{\boldsymbol{w}} & \boldsymbol{w}^{\prime} \hat{E}(\boldsymbol{g})-\frac{1}{2} \boldsymbol{w}^{\prime} \hat{\boldsymbol{\Sigma}} \boldsymbol{w} \\
\text { s.t. } & \boldsymbol{w}^{\prime} \boldsymbol{\imath}=1 \quad l_{i} \leq w_{i} \leq u_{i}, \quad i=1, \cdots, N
\end{array}
$$

where $l_{i}$ and $u_{i}$ are lower and upper bounds on asset $i$ weight.

This procedure takes care of the problem identified in the previous section. The Black Litterman alpha is derived from an equilibrium model as well as from a prior. Information on the mean returns is not discarded, removing the potential for the type of loss identified in the previous section.

This procedure, however, introduces a new type of problem. It is based on two models that are contradictory to each other. The prior is built on the belief that a factor model is the underlying data generating process, while the likelihood is based on the belief that a CAPM is the underlying data generating process. Both beliefs cannot be true at the same time. As the procedure is based on two beliefs, the procedure cannot be optimal regardless of which belief is accurate. ${ }^{29}$ In the following subsection, we will show the magnitude of the loss arising from adopting this procedure.

\subsection{The Cost of Using Contradictory Models}

\subsubsection{The Model and the View}

We will assume that the factor model in Equation (27) is the true data generating process, and we will show that using the FFK procedure creates a loss. Our argument is independent of what the true model is.

In the previous section, we already discussed the factor model in Equation (27), a prior for this model, and the resulting predictive mean and the variance. The only difference is that in Equation (27) we have $\boldsymbol{\Sigma}_{e}$ instead of $\frac{1}{h} V_{e}$. We will also make one modification to the prior

${ }^{28} \hat{E}(\boldsymbol{g})$ is the posterior mean of the stock return, as it is the posterior mean of the mean returns.

${ }^{29}$ Meucci's [9] extension of BL has a similar feature. That is, it is based on two inconsistent models. Meucci, however, provides an alternative, more appealing justification of his approach based on the idea of relative entropy. Thus, our criticism has limited relevance to Meucci's approach. 
in this section. We will assume that the investor does not have any prior opinion. This modification not only makes the calculation much simpler, but it also makes our comparison more relevant. If we suppose that the investor who follows the FFK procedure has a certain prior opinion, then this investor is making twice as many mistakes. The first mistake is ignoring his prior opinion, and the second mistake is not using the factor model exclusively. Thus, by assuming that the investor does not have any prior opinion, we are eliminating one possible mistake that the investor could commit by following the FFK procedure.

Formally, the non-informative prior can be expressed as

$$
\begin{aligned}
& \boldsymbol{f} \mid h \sim N\left(\boldsymbol{m}_{f}, \frac{1}{h} \boldsymbol{V}_{f}\right), \quad \boldsymbol{V}_{f} \rightarrow \infty \\
& h \sim G(p, q), \quad q \rightarrow 0
\end{aligned}
$$

This is rather intuitive as it says that the noninformative prior is just the prior distribution of the factor premium as the variance of the factor premium converges to infinity. The formula for the posterior must be modified as well. The new formulae are: ${ }^{30}$

$$
\begin{aligned}
& \tilde{E}(\boldsymbol{f})=\hat{\hat{\boldsymbol{f}}} \\
& \tilde{V}(\boldsymbol{f})=\frac{\boldsymbol{S}_{e}}{N T-2} \boldsymbol{S}_{B}^{-1} \\
& \tilde{V}\left(\boldsymbol{e}_{T+1}\right)=\frac{\boldsymbol{S}_{e}}{N T} \hat{\boldsymbol{\Sigma}}_{e}
\end{aligned}
$$

The predictive mean and the predictive variance-covariance matrix of the returns are then:

$$
\begin{aligned}
\tilde{E}\left(\boldsymbol{r}_{T+1}\right) & =\boldsymbol{B}_{T} \hat{\hat{\boldsymbol{f}}} \\
\tilde{V}\left(\boldsymbol{r}_{T+1}\right) & =\frac{\boldsymbol{S}_{e}}{N T-2} \boldsymbol{B}_{T} \boldsymbol{S}_{B}^{-1} \boldsymbol{B}_{T}^{\prime}+\frac{\boldsymbol{S}_{e}}{N T} \hat{\boldsymbol{\Sigma}}_{e}
\end{aligned}
$$

\subsubsection{An Analytic Formula for Loss}

Consider two investors. One investor, called the FFK investor, follows the FFK procedure as described in the previous subsection. The other investor, called the RTL investor, makes rational decisions based on the factor model.

The RTL investor's portfolio can be specified as

$$
\begin{aligned}
& \boldsymbol{w}_{R T L}=\operatorname{argmax}_{w} \quad \boldsymbol{w}^{\prime} \tilde{E}\left(\boldsymbol{r}_{T+1}\right)-\frac{1}{2} \boldsymbol{w}^{\prime} \tilde{V}\left(\boldsymbol{r}_{T+1}\right) \boldsymbol{w} \\
& \text { s.t. } \quad \boldsymbol{w}^{\prime} \boldsymbol{\iota}=1 \quad l_{i} \leq w_{i} \leq u_{i}, \quad i=1, \cdots, N
\end{aligned}
$$

and the RTL investor's utility can be written as

$$
U_{R T L}=\boldsymbol{w}_{R T L}^{\prime} \tilde{E}\left(\boldsymbol{r}_{T+1}\right)-\frac{1}{2} \boldsymbol{w}_{R T L}^{\prime} \tilde{V}\left(\boldsymbol{r}_{T+1}\right) \boldsymbol{w}_{R T L}
$$

${ }^{30} \boldsymbol{S}_{e}, \boldsymbol{S}_{B}, \hat{\hat{\boldsymbol{f}}}, \hat{\boldsymbol{\Sigma}}_{e}$ need to be modified as well.
The FFK investor's portfolio can be specified as

$$
\begin{aligned}
& \boldsymbol{w}_{F F K}=\operatorname{argmax}_{w} \quad \boldsymbol{w}^{\prime} \hat{E}(\boldsymbol{g})-\frac{1}{2} \boldsymbol{w}^{\prime} \hat{\boldsymbol{\Sigma}} \boldsymbol{w} \\
& \text { s.t. } \quad \boldsymbol{w}^{\prime} \boldsymbol{\imath}=1 \quad l_{i} \leq w_{i} \leq u_{i}, i=1, \cdots, N
\end{aligned}
$$

and the FFK investor's utility can be written as

$$
U_{F F K}=\boldsymbol{w}_{F F K}^{\prime} \tilde{E}\left(\boldsymbol{r}_{T+1}\right)-\frac{1}{2} \boldsymbol{w}_{F F K}^{\prime} \tilde{V}\left(\boldsymbol{r}_{T+1}\right) \boldsymbol{w}_{F F K}
$$

As in the previous section, we calculate the loss analytically for the simplest case, i.e. the case where there are no constraints in the optimization. If there are no constraints in the optimization, the RTL investor's portfolio weight and his utility are identical to those presented in the previous section, i.e.

$$
\begin{gathered}
\boldsymbol{w}_{R T L}=\tilde{V}\left(\boldsymbol{r}_{T+1}\right)^{-1} \tilde{E}\left(\boldsymbol{r}_{T+1}\right) \\
U_{R T L}=\frac{1}{2} \tilde{E}\left(\boldsymbol{r}_{T+1}^{\prime}\right) \tilde{V}\left(\boldsymbol{r}_{T+1}\right)^{-1} \tilde{E}\left(\boldsymbol{r}_{T+1}\right)
\end{gathered}
$$

The FFK investor's portfolio weight, on the other hand, is:

$$
\boldsymbol{w}_{F K K}=\hat{\boldsymbol{\Sigma}}^{-1} \hat{E}(\boldsymbol{g})
$$

For simplicity, let us assume that the FFK investor's estimate of the variance-covariance matrix $\tilde{V}\left(\boldsymbol{r}_{T+1}\right)$ is not very different from the predictive variance-covariance matrix $\tilde{V}\left(\boldsymbol{r}_{T+1}\right)$ of the RTL investor. ${ }^{31}$ Then we could write

$$
\boldsymbol{w}_{F K K} \approx \boldsymbol{w}_{R T L}+\tilde{V}\left(\boldsymbol{r}_{T+1}\right)^{-1}\left[\hat{E}(\boldsymbol{g})-\tilde{E}\left(\boldsymbol{r}_{T+1}\right)\right]
$$

and

$$
\begin{aligned}
& U_{F F K} \\
& \approx U_{R T L}-\frac{1}{2}\left[\hat{E}(\boldsymbol{g})-\tilde{E}\left(\boldsymbol{r}_{T+1}\right)\right]^{\prime} \tilde{V}\left(\boldsymbol{r}_{T+1}\right)^{-1}\left[\hat{E}(\boldsymbol{g})-\tilde{E}\left(\boldsymbol{r}_{T+1}\right)\right]
\end{aligned}
$$

Thus, the loss is approximately quadratic in the difference between the FFK investor's posterior mean and the RTL investor's posterior mean.

Let us consider the difference between the FFK investor's posterior mean and the RTL investor's posterior mean further. Assuming that the difference between the OLS estimator $\hat{\boldsymbol{f}}$ and the GLS estimator $\hat{\boldsymbol{f}}$ is not $\operatorname{large} e^{32}$, one can write

$$
\hat{E}(\boldsymbol{g})-\tilde{E}\left(\boldsymbol{r}_{T+1}\right) \approx\left(\hat{\boldsymbol{\Sigma}}_{e}^{-1}+\frac{1}{\tau} \hat{\boldsymbol{\Sigma}}^{-1}\right)^{-1} \frac{1}{\tau} \hat{\boldsymbol{\Sigma}}^{-1}\left(\boldsymbol{\pi}-\boldsymbol{B}_{T} \hat{\hat{\boldsymbol{f}}}\right)
$$

Thus, the loss depends on the difference between the

\footnotetext{
${ }^{31}$ That is, we are assuming that the FFK investor is using the best estimator of the variance-covariance matrix. This assumption is of course more favorable to the FFK investor.

${ }^{32}$ This happens when the error covariance is close to an identity matrix, i.e. homeskedatic and no serial correlation.
} 
multifactor model estimates and the CAPM estimates. This is a rather obvious result, considering that the very source of the problem was the use of the CAPM, which is not the correct model in our example.

\subsubsection{Investor Simulations}

We follow the procedure identical to that of the previous section to numerically calculate the magnitude of the loss. ${ }^{33}$ Tables $\mathbf{2}$ and $\mathbf{3}$ summarize the simulation results. The actual distribution of losses for the FFK investor for 1000 simulations is shown in Figure 2. The mean loss for the FFK investor is between 0.09 and 0.12 , while the median loss is between 0.06 and 0.10 . As before, we might interpret this loss as a reduction in the expected return of the investor. A conservative estimate would be a loss of around $0.09 \%$ in expected return per month or $1.08 \%$ on an annualized basis.

One may notice that the loss of the FFK investor is somewhat smaller than the loss of the JLZ investor. This is what we could have expected. The loss of the JLZ investor is a quadratic function of the error in the mean estimate, while the loss of the FFK investor is a linear function of the difference in mean estimates of the two models.

\section{Using the Black-Litterman Approach as a Reverse Optimization Technique}

Practitioners often use the Black-Litterman approach as a tool to extract implied expected returns for a given portfolio weight vector. Step 3 of the JLZ procedure discussed in Section 2 of this paper is an example of this.

Given a portfolio weight vector $\boldsymbol{w}_{0}$, one can derive the implied expected return by assuming that the portfo-

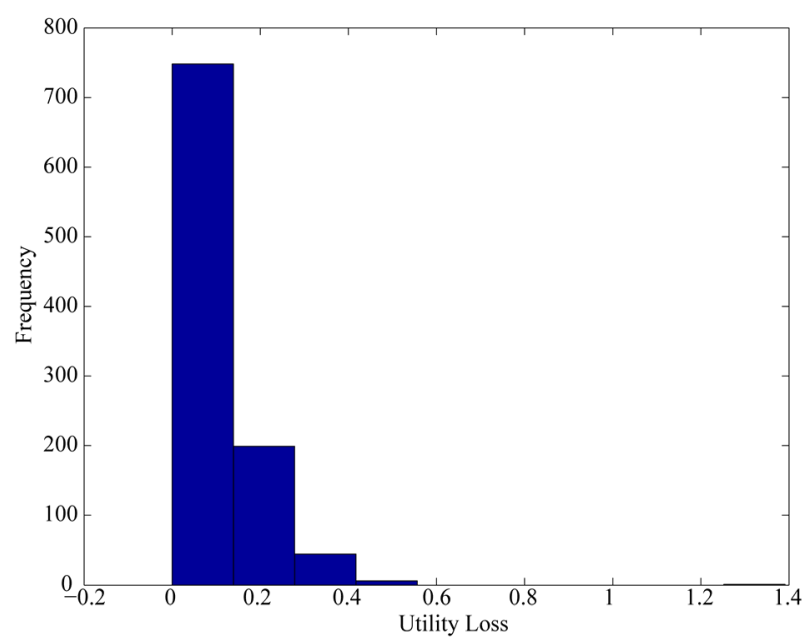

Figure 2. Utility Losses from 1000 Simulations for the FFK Investor.

\footnotetext{
${ }^{33}$ See the appendix available at

http://papers.ssrn.com/sol3/papers.cfm?abstract id=2191278 for more details.
}

lio weight vector is a solution to the following optimization problem:

$$
\boldsymbol{w}_{0}=\operatorname{argmax}_{\boldsymbol{w}} \quad \boldsymbol{w}^{\prime} \boldsymbol{\alpha}-\frac{1}{2} \boldsymbol{w}^{\prime} \hat{\boldsymbol{\Sigma}} \boldsymbol{w}
$$

As we have shown in Section 2, the solution is given as

$$
\alpha=\Sigma w_{0}
$$

where $\boldsymbol{\Sigma}$ is the variance-covariance matrix of returns.

One aspect that is often ignored in the "reverse optimization" is that the validity of the implied expected return depends on the validity of the variance-covariance matrix used. ${ }^{34}$ To the extent that the variance-covariance matrix includes an error, the implied expected return also includes the error.

Let $\boldsymbol{\Sigma}_{0}$ be the true variance-covariance matrix. Let $\boldsymbol{\Sigma}_{1}$ be our estimate of the variance-covariance matrix. Then the error in the implied expected return is $\left(\boldsymbol{\Sigma}_{1}-\boldsymbol{\Sigma}_{0}\right) \boldsymbol{w}_{0}$.

To have a more intuitive sense of the magnitude of the error, we focus on the implied market return, which is $\boldsymbol{w}_{M}^{\prime}\left(\boldsymbol{\Sigma}_{1}-\boldsymbol{\Sigma}_{0}\right) \boldsymbol{w}_{0}$, where $\boldsymbol{w}_{M}$ is the market capitalization weight. We present typical values of this bias in Table 4, which were calculated from a simulation. ${ }^{35}$ When $\boldsymbol{w}_{0}$ reflects a value weight portfolio , the $80 \%$ range of the error is from -2.6 to 2.75 . That is, excluding the $20 \%$ worst cases, the error in the expected monthly market return can be as high as $2.75 \%$. When $\boldsymbol{w}_{0}$ reflects an equal-weighted portfolio, the same error can be as high as $3.52 \%$. This magnitude of the error can be compared to the long-term average return of the market, which is around $2 \%$. This is a large error in relative terms.

\section{Conclusions}

The Black-Litterman model has been used extensively in

Table 4. Bias in the Implied Expected Monthly Market Returns.

\begin{tabular}{cccccc}
\hline Type & Mean & SD & 10th Pct & Med & 90th Pct \\
\hline VW & 0.001 & 2.09 & -2.61 & 0.11 & 2.75 \\
EW & 0.010 & 2.68 & -3.33 & -0.13 & 3.52 \\
\hline
\end{tabular}

Note: VW represents the reverse optimization bias for value-weighted portfolio, while EV represents the reverse optimization bias for an equalweighted portfolio in percentage terms.

\footnotetext{
${ }^{34}$ That one recovers the expected return vector rather than the variance-covariance matrix from the reverse optimization may reflect the belief that estimating the variance-covariance matrix is easier than estimating the expected return vector. Even if such a belief is reasonable, one shouldn't forget that the implied expected return is an estimate.

${ }^{35}$ See the appendix available at

http://papers.ssrn.com/sol3/papers.cfm?abstract_id=2191278 for details of the simulation.
} 
asset allocation and in recent years, a number of people have proposed applications of it to areas such as trading and quantitative portfolio management. Some of these methods have necessitated a transformation or adaptation of the Black-Litterman model to the specific application. Unfortunately, these transformations could be associated with unintended consequences. In this paper, we discuss three applications of the Black Litterman model that result in unnecessary costs to the investor.

The first type of application creates a portfolio out of a prior and an estimate of the variance-covariance matrix, but fails to utilize the mean estimate. Not using the mean estimate amounts to ignoring a valuable piece of information present in the data. Our conservative estimate of the loss from neglecting the mean is about a $1 \%$ reduction in expected annual returns. Although it is well known that means are not estimated as reliably as variance and covariances, ignoring the mean estimate cannot be an optimal solution. For even if the mean estimate is not extremely precise, it is still better to use this estimate than to entirely ignore it.

The second type of application creates a portfolio out of two conflicting models of security returns, e.g. the CAPM and a multi-factor model. Assuming that the multi-factor model is the true model of stock returns, we estimate the loss to an investor that chooses to combine both a CAPM model and a multi-factor model in his portfolio construction. We find the magnitude of this loss to be around a $1 \%$ reduction in expected annual returns. One might justify the use of two models if the portfolio manager has no idea regarding which model is more likely to be true. In most practical situations, it would be unlikely that the portfolio manager or analyst did not have a clear idea about which model they believed represented the behavior of stock returns. Thus, it makes it hard to justify using two contradictory models of stock returns.

The third application is the so-called reverse optimization technique. That is, practitioners often use the weights of an index and reverse optimize to obtain the implied expected returns of the market. Since the variance-covariance matrix is estimated with error, the implied expected returns of such a procedure will also be estimated with error. We quantify the magnitude of the errors associated with this technique. We found this error to be quite high, in some cases as high as 3.5\% per month and much higher if the original benchmark was an equal-weighted benchmark. This brings into question how useful this reverse optimization technique really is.

No doubt the Black-Litterman model brought a new tool to aid asset allocators, portfolio managers, and traders with the construction of optimal portfolios. In particular, it provided a theoretical framework upon which an investor's prior views about asset markets or stocks could be combined with the actual historical data in order to construct optimal investments. The main application of the Black-Litterman model has been to asset allocation across broad asset classes. Recent research has tried to integrate the Black-Litterman model in the trading framework and the quantitative equity portfolio framework. These advances are inspiring as they improve the tool set for quantitative managers, unfortunately some of the applications have side effects which we must be aware of. In this paper, we have discussed some of the potential side effects of these applications of the Black-Litterman model. Our estimates of the losses associated with these side effects are large from a portfolio management perspective. We also suggest a straightforward way to apply the Black-Litterman model without this bias in the portfolio construction process.

\section{REFERENCES}

[1] G. L. He and R. Litterman, "The Intuition Behind the Black-Litterman Model Portfolios," Goldman Sachs Working Paper, 1999.

[2] F. Black and R. Litterman, "Global Portfolio Optimization," Financial Analysts Journal, Vol. 48, No. 5, 1992, pp. 28-43. doi:10.2469/faj.v48.n5.28

[3] L. B. Chincarini and D. Kim, "Quantitative Equity Portfolio Management," McGraw-Hill, New York, 2006.

[4] R. C. Jones, T. Lim and P. J. Zangari. "The Black-Litterman Model for Structured Equity Portfolios," Journal of Portfolio Management, Vol. 33, No. 2, 2007, pp. 24-43. doi:10.3905/jpm.2007.674791

[5] F. J. Fabozzi, S. M. Focardi and P. Kolm, "Incorporating Trading Strategies into the Black-Litterman Framework," Journal of Trading, Vol. 1, No. 2, 2006, pp. 28-37.

[6] S. Satchell and A. Scowcroft, "A Demystification of the Black-Litterman Model: Managing Quantitative and Traditional Portfolio Construction," Journal of Asset Management, Vol. 1, 2000, pp. 138-150. doi:10.1057/palgrave.jam.2240011

[7] A. Zellner, "An Introduction to Bayesian Inference in Econometrics," John-Wiley and Sons, Hoboken, 1971.

[8] G. Koop, "Bayesian Econometrics," John-Wiley and Sons, Hoboken, 2003.

[9] A. Meucci, "Fully Flexible Views: Theory and Practice," Risk Magazine, Vol. 21, No. 10, 2008, pp. 97-102. 